\title{
THE DRONE - A METHODOLOGICAL TOOL, FOR GENERATING BASE LAYERS IN GIS
}

DOI: http://dx.doi.org/10.18509/GBP.2018.56

UDC: 004:528.8/.9]:623.746.2-519

\author{
Enea Andrei ${ }^{1,2}$ \\ Iosub Marina ${ }^{1,2}$ \\ Stoleriu Cristian Constantin ${ }^{1}$ \\ Ursu Adrian ${ }^{1}$ \\ Romanescu Gheorghe ${ }^{1}$ \\ ${ }^{1}$ Alexandru Ioan Cuza University of Iasi, Faculty of Geography and Geology, Department of \\ Geography, Bd. Carol I 20A, 700505, Iasi, Romania \\ ${ }^{2}$ Integrated Center of Environmental Science Studies in the North Eastern Region-CERNESIM, \\ 11 Carol I, 700506,"Alexandru Ioan Cuza" University of Iasi, Romania
}

\begin{abstract}
One of the latest paradigms of today's interdisciplinary studies in geosciences, consists of the implementation of the newest, most accurate, and relevant datasets available, in order to emphasize the appearance, causality or effects of different phenomena, which interfere with humans. Therefore, there is a permanent strive for data, relevant in geographical analysis, which is highly accurate, and also cost-effective. Due to the recent developments in UAV technology, and lowering of production costs, drones have been integrated into methodological workflows all around the world, in numerous fields, ranging from habitat delineation, to geomorphologic mapping. Most such studies use either a digital surface model (DSM) or ortophoto imagery generated from drone aerial images. Also, Structure From Motion algorithms (SFM) have been highly developed recently, into detecting ever more complicated shapes and objects. This means that the drone has turned into an indispensable tool for generating base layers used in any GIS-based study, because it generates fast, high accuracy, repeatable, on demand data sets. This paper intends to reveal a methodological approach towards generating the two, most important raster layers for the majority of spatial analyses: the digital surface model/digital elevation model, and the ortophoto, respectively.
\end{abstract}

Keywords: drone, DSM, GIS, UAV

\section{INTRODUCTION}

Research in geosciences is highly dependent on input data, such as spatial data, numerical data in table form, graphs or various form of imagery. There are several aspects of data parameters, that are extremely relevant in every analysis, which directly determine the quality of the final results. Some of the most important data parameters are frequency, continuity, size of data sets, resolution, and quality. Depending on the domain of research, the data required is slightly different, but it reveals a spatial reality, relevant for the specific study. One of the most common issues researchers face is the lack of data, poor spatial resolution, or non-synchronous data with the period of time that is relevant for a study. During the last years, technology has advanced sufficiently enough, to offer massscale solutions for spatial data collection, starting from world-wide satellite coverage of 
images in visible and spectral ranges, up to drone-acquired imagery that offer several types of useful layers [1], [2].

The most relevant layers that can be generated from drone platforms are Digital Elevation Models (DEMs'), ortophoto images, spectral images, and Light Detection and Ranging (LIDAR) data [3-6]. Drones, also named UAVs (Unmanned Aerial Vehicles), vary greatly in size and load capacities, the more sophisticated sensors (spectral or LIDAR) requiring larger carrying platforms.

Geographic Information Systems use the drone-derived data layers to analyse natural or anthropic processes, or phenomena, in a wide range of fields of interests, such as hydrology, geomorphology, risk management, urban planning, civil engineering, search and rescue, forestry, biology, archaeology etc. [7-12].

Also, there are specific software, oriented towards calibrating, managing, georeferencing and transforming drone images into the aforementioned digital elevation models and ortophoto layers. These range from open source solutions (Visual SFM, CMVS and CMPMVS), up to proprietary software, such as Agisoft Photoscan Professional (www.agisoft.com), or Pix4D Mapper (www.pix4d.com). Generally, the paid software offers a much more complete solution, and a more user-friendly interface, for transforming drone imagery into GIS layers [13], [14].

\section{METHODS AND WORKFLOW}

There are several stages before, during, and after the data acquisition flights, undergone by drone pilots, for recording the aerial images. For the purpose of this study, the following methodological discussions concern the generation of digital elevation models and ortophoto imagery [15-17].

Firstly, the flight plan has to be conceived, with several aspects in mind. The flight route has to successfully overlap the images on over a minimum area of interest (usually 60$80 \%$ ). The flight area has to be checked for any potential obstacles, and a permit is required from local and aeronautical authorities, in order to be in conformity with national flight regulations. Also, depending on the study regions' morphology and local particularities, extra images are required to be recorded, for full coverage from all angles (mostly for complex object volumetric shapes, such as river dams, irregular buildings, landscape forms with underslung elements etc.).

Secondly, before the image recording flight, high-contrast GPS markers are deployed in the field, as close to the study area extremities, as possible, and high precision coordinates for each marker are recorded (note: a minimum of 4 markers is mandatory, but 10 or more markers are recommended). After a preliminary GPS calibration, the flight itself requires a sufficient number of overlapping images, from as many necessary angles, as needed, for generating a complete model.

There are several factors that highly influence the flight and accuracy of the final products. Some of the more important are, as follows:

- Image resolution: the higher the resolution of the aerial images, the more precise the accuracy of the final layers will be (a higher resolution is not only useful in generating finer detail layers, but also improves the correctness of the results)

- Camera calibration: refers to the removal of geometric distortions, the drone images requiring preprocessing

- Camera orientation: it is extremely important to guarantee that the UAV camera is oriented in the direction of the study area. This issue is especially relevant for 
completely autonomous flying drones, that need to be given the camera orientation input, to assure that the subject area is in frame, during unassisted flights

- Angle between images: to obtain the best results, each spatial point in the study area must be recorded in as many images as possible, for the $3 \mathrm{D}$ point cloud algorithm to generate valid, accurate results. There are numerous objects that cannot be completely described in point clouds, only by combining aerial images taken exactly from vertical orientations; they require the drone to fly lower to the ground, while recording images from lower angles of the aforementioned particular objects (Fig. 1)

- Image sufficiency: to reduce error occurrence, a larger number of images is recommended to be recorded. Certain drone platforms have a landing gear that occasionally interferes with the images that are being recorded (especially under high wind speeds, or high drone speeds), which need to be filtered out during the cloud generation stages, to avoid artefacts being introduced in the analysis

- Relative flight altitude: the further away the drone is, in regard to the topography of the land, the lower the spatial resolution of the final results. Pixel size is, therefore, directly influenced by flight altitude

- Atmospheric conditions: a number of weather factors influence the process of collecting drone images, but also their quality. Light plays the most important role, which influences the results. Rich, diffuse light will generate different looking layers, compared to sunny conditions. Also, the presence of foggy conditions limits the clarity and contrast of the images. From a technical point of view, the presence of rain, humidity and wind, induces the highest degree of risk, creating condensation on the lens, or the electronic circuits, possibly inducing short circuiting, resulting in potential complete malfunction of the drone

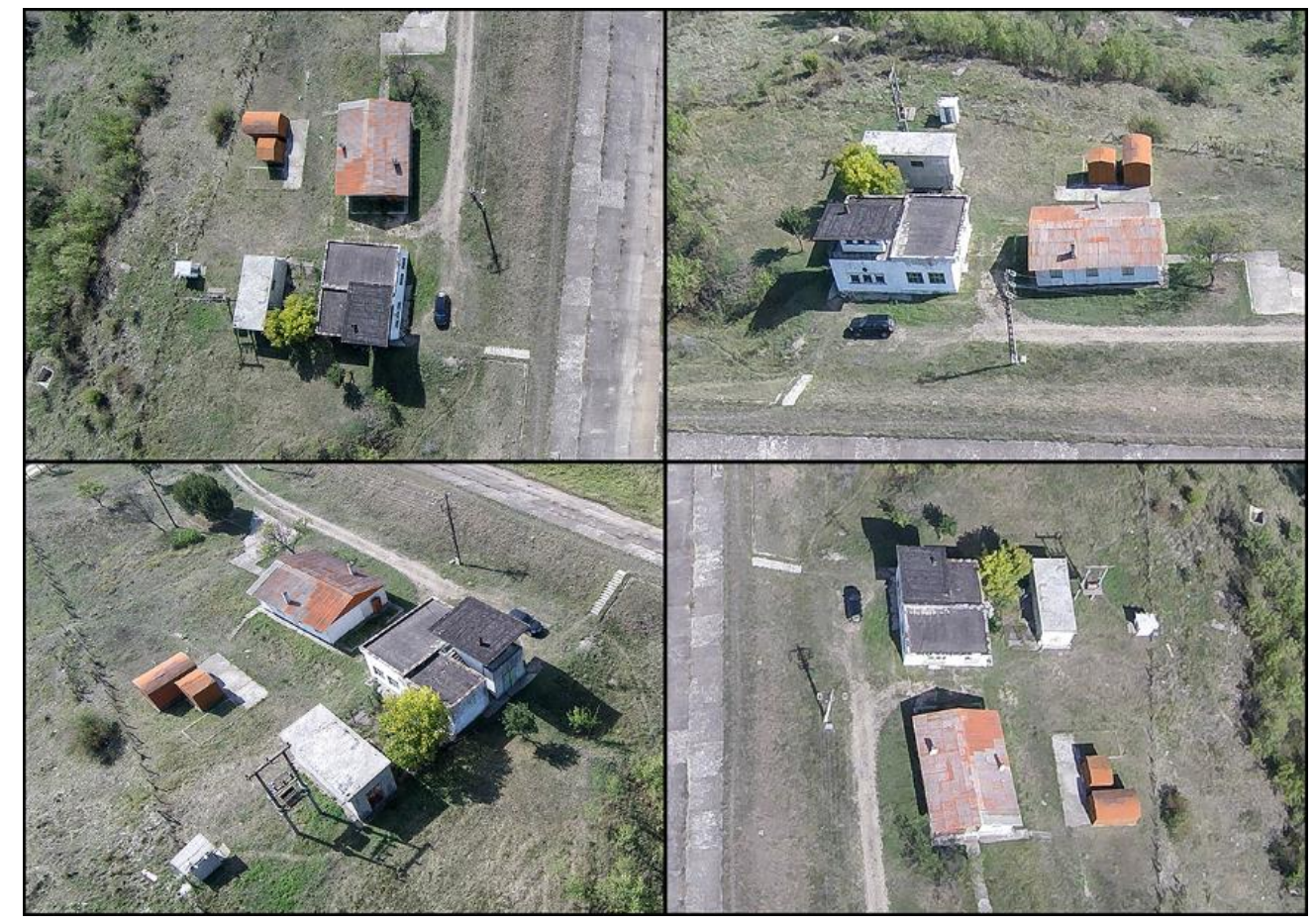

Figure 1. Example of different angles in drone-acquired imagery, for the same interest area, during a single flight

As mentioned, following the flight, the images are downloaded, and imported into a dedicated image processing software. For the Structure From Motion (SFM) software to 
correctly tie common points on different aerial images, the first preprocessing step would be to correct the entire set of images of geometric distortions. This is usually done, by applying a camera/lens profile, that has been precisely factory calculated, in order to remove any distortions in the images.

During the processing stage, there are multiple workflow steps. From one software, to another, there are variances and small differences, during this process, but for the purpose of conceptual understanding, Agisoft Photoscan Professional will be used as an example, to offer a concrete methodological recommendation.

The calibrated images need to be imported, proceeding with an alignment function, which analyses all the images in the data set. The first steps are particularly important, as they define the resolution and accuracy of the final 3D model. During this step, the accuracy of the alignment can be chosen and key points and tie points limits can be adjusted. While it is generally recommended to select high accuracy values, with high input parameters, these options can be associated with slow render speeds, or even the possibility of higher error occurrence. During this stage, the images are correctly placed in three-dimensional space, (Fig. 2), and Structure From Motion algorithms generate points, based on them, in order to generate the sparse point cloud.

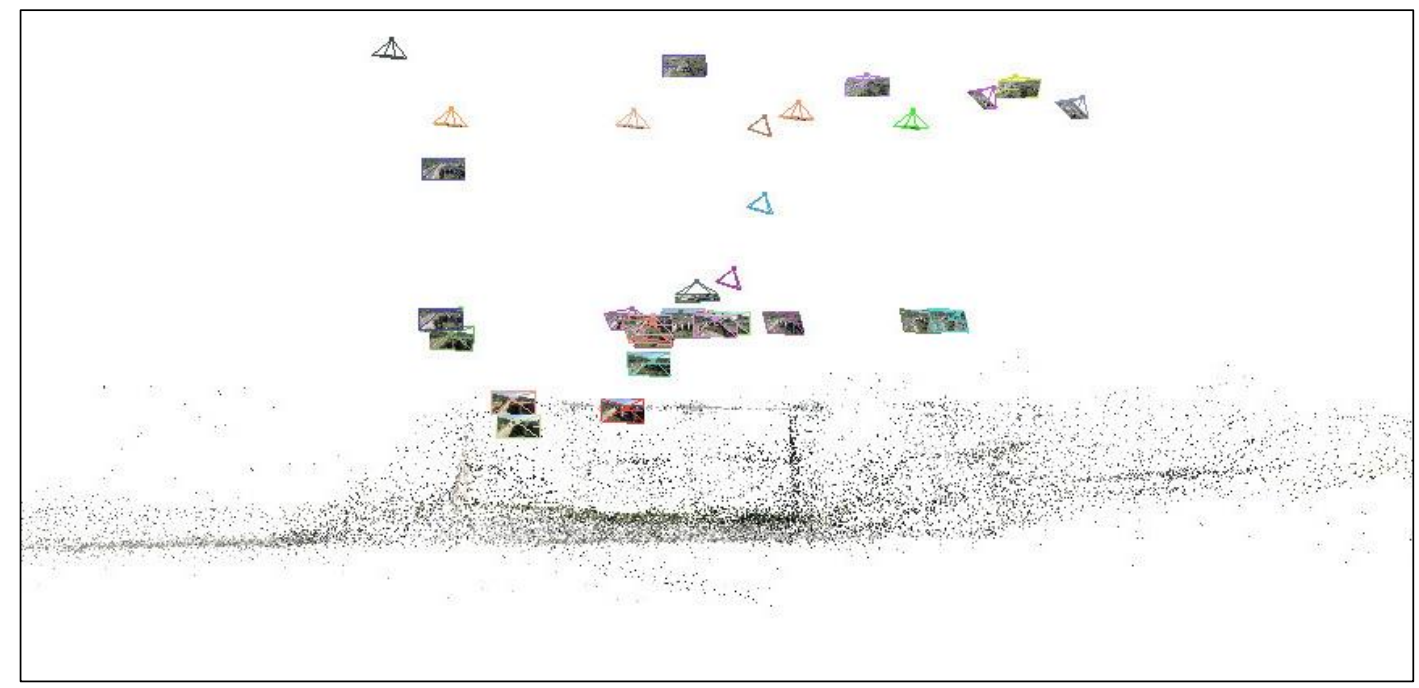

Figure 2. Point cloud generated using Structure From Motion techniques, with the corresponding aerial images it was generated from, and their relative position, in three dimensional space. Screen capture of

Belci dam point cloud, generated in open source software Visual SFM (http://ccwu.me/vsfm/)

The next step is to build a dens point cloud, in order to increase the magnitude of detail, from which a mesh layer can be created. This step is highly important, because the resulting Digital Surface Model (DSM), that is recommended to be saved in a TIFF elevation format, will be directly dependent to the cloud density of the dense cloud file, and its' corresponding mesh layer. Following this, the mesh and texture layers are computed.

Georeferencing the model is a particularly important stage, due to the future usage in GIS software. Its' importance is given by the fact that low GSD values are irrelevant, if there isn't a precise spatial placement, with correct georeferencing procedures. Classically, the georeference stage would consist in associating attributes (geographical coordinate points), recorded in the field stage, to the marker locations on the map, through manual input in the marker panel after the layer generation process is finished (Fig. 3). 


\begin{tabular}{|l|rrr|}
\hline Markers & Easting & Northing & Altitude \\
$\square \mathbb{P}$ point 1 & 636170.877000 & 533506.425000 & 208.000000 \\
$\square \mathbb{P}$ point 2 & 635857.724000 & 533703.572000 & 208.000000 \\
$\square \mathbb{P}$ point 3 & 636170.389000 & 533676.759000 & 206.000000 \\
$\square \mathbb{P}$ point 4 & 635928.556000 & 533487.314000 & 207.900000 \\
$\square \mathbb{P}$ point 5 & 636074.626000 & 533348.381000 & 219.500000 \\
$\square \mathbb{P}$ point 6 & 636059.286000 & 533539.524000 & 213.500000 \\
$\square \mathbb{P}$ point 7 & 636043.593000 & 533783.286000 & 207.000000 \\
$\square \mathbb{P}$ point 8 & 636064.673000 & 533457.870000 & 216.600000 \\
Total Error & & & \\
\hline
\end{tabular}

Figure 3. Example of georeference point table, as input in Agisoft Photoscan Professional, with altitudine values (expressed in meters)

The most modern UAV platforms can automatically insert metadata into each image that is being recorded during survey flights, consisting in geographical coordinates and even flight altitude. This approach is particularly useful in areas where marker deployment is extremely difficult, or impossible, and georeferencing can be done automatically in the software, without the need of manual input for geographical coordinates. Alternatively, georeferencing through correspondence points, taken from maps with similar spatial resolutions can be performed, in these situations, when coordinate markers cannot be physically deployed.

After correctly inserting the georeference, the DSM can be exported, as well as the correlated ortophoto file. In multiple use case scenarios, this model is not adequate, because it includes the non-topographical elements, such as buildings, or vegetation, but there are cases where this is required (studies of forest extent, urban viewshed analysis, volumetric calculations etc.) (Fig. 4).
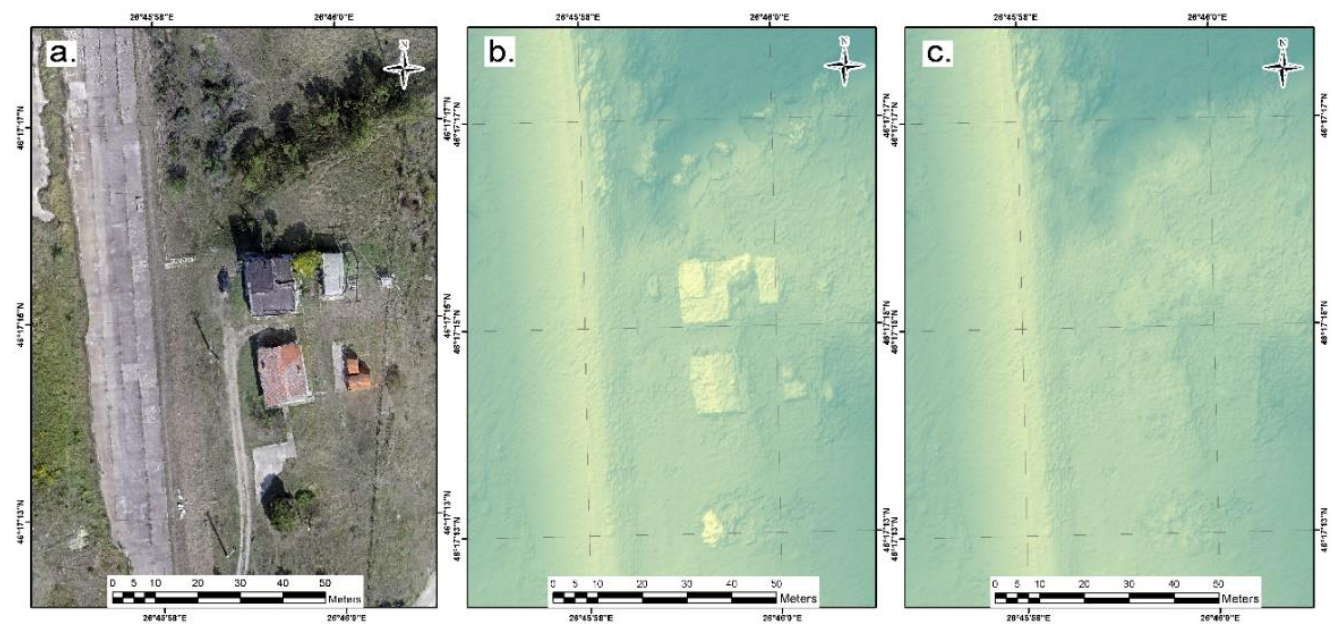

Figure 4. Different drone-generated layers, used in GIS analysis: a. An ortophoto image with several, different contained elements; b. A Digital Surface Model (DSM) with constructions and vegetation as elements with relative elevation values; c. A Digital Terrain Model (DTM), generated by removing all the spatial elements from the DSM (such as buildings or trees), except for the topographic surface. This spatial example is located in the proximity of the former Belci dam, Tazlau river, Romania, and the layers were generated from drone-aquired images, through Structure From Motion (SFM) techniques, in Agisoft

Photoscan Professional

Therefore, corrections need to be made, in order to obtain the Digital Terrain Model (DTM). There are several methods of approaching this, but the most precise ones involve supplementary, in-field measurements, mostly under the trees, where vegetation height is 
very difficult to be assessed after field stages. This involves recording topography values with a theodolite, or a total station, and generating altitude points, in the areas where vegetation is abundant. On the workstation, firstly, the surface model undergoes a contouring or altitude point extraction process (either way, it is necessary for the resulting layers to be vectors with altitude attribute values) at a very high precision (for example, contour lines equidistance set to under 1 meter). Next, the regions with non-topographical elements are isolated and deleted, so that only the vector elements which depict topographical altitude remain. In the newly created hollow regions, the topographic field measurements are inserted, and the new model (a DTM) can be interpolated, while maintaining a high spatial resolution.

Using an inverse logic, by isolating vegetation, and integrating solely this layer into a different type of analysis, and comparing it to satellite imagery, or topographical data, several studies can be performed (tree height morphometry, land cover evolution etc.). Also, by analyzing several temporal sets of elevation data, precision morphometric studies can be generated, such as landfill waste deposits, construction sites, biomass evolution, geomorphological erosion in gullies, or even surface erosion dynamics (where the elevation models can be generated to a very high spatial resolution), river embankment evolution, river meandering amplitude, sedimentation processes, habitat expansion/contraction etc. There are several advantages and disadvantages for using a drone for generating DSM, DTM and ortophoto models.

The main advantages involve the price of data acquisition, which is very reduced, considering drone prices have dropped significantly up to present date; the frequency with which data can be collected, and elevation models (as well as ortophoto imagery) can be created, especially in evolution analyses for fast-paced processes or phenomena (in geomorphology, agriculture, hydrology, biology, forestry etc.). This means that the lack of daily, under 1-meter resolution satellite images can now be countered with daily, weekly, monthly, seasonal or annual GIS layers, with high resolution. Also, the temporal advantages for drone generated layers include the possibility to fly and create on-demand models, at any time, in unpredictable situations (floods, wildfires, landslides, accidents or other risk phenomena). Another benefit of using these type of models is the ability to analyze areas which do not offer the possibility for physical presence, the non-invasive character of a drone being, in many situations, the only possibility to collect data (in very hazardous environments, in strictly protected natural regions, or in unreachable areas). Also, the data resolution is extremely high, such models being very suitable for detailed studies, which could otherwise not be possible on coarse-resolution satellite images.

There are drawbacks, as well, but mostly of technical origin, the greatest issue concerning the flight autonomy on one battery. The vast majority of drones are currently batterypowered, and even the most efficient ones only slightly surpass a flight time of 30 minutes. Until current battery technologies will change, to a more power efficient alternative, drones with vertical take-off and landing will only be useful on smaller study areas. There are alternatives with remotely operated planes, but the lack of hover capabilities make them unusable in certain, low-altitude flight operations. Also, legal aspects play an important role, flight authorisations or licenses being required in numerous countries. 


\section{CONCLUSIONS}

The ever increasing research standards of the modern, scientific community faces, involves searching for alternative data collection and generation methodologies, which comply with high accuracy demands in all fields of geosciences. Despite the complex methodological workflows that can be addressed for creating digital surface or terrain elevation models, results in numerous fields whose analysis's are fundamentally based in GIS software, have proven to replicate and improve the previously accepted standards, such as satellite or ortophoto imagery recorded by using airplanes.

There are numerous advantages of incorporating drone platforms into scientific research, ranging from high resolution for any geospatial application, temporal repeatability at user discretion, non-invasive remote operation in difficult to reach study sites, up to reduced flight costs and ease of use. Structure From Motion algorithms are used to generate Digital Surface Models, from which Digital Terrain Models can be derived, for a wide variety of fields of research: hydrology, geomorphology, risk management, urban planning, civil engineering, search and rescue, forestry, biology, archaeology, or other natural or anthropic processes or phenomena. Therefore, unmanned aerial vehicles have proven to be a modern, indispensable tool in generating GIS layers, of great importance, resolution, and repeatability, at demand, and with extreme ease.

\section{ACKNOWLEDGEMENT}

This work was financially supported by the Department of Geography from the "Alexandru Ioan Cuza" University, of Iasi, and the infrastructure was provided through the POSCCE-O 2.2.1, SMIS-CSNR 13984-901, No. 257/28.09.2010 Project, CERNESIM.

\section{REFERENCES}

[1] Harwin S. \& Lucieer A. Assessing the Accuracy of Georeferenced Point Clouds Produced via Multi-View Stereopsis from Unmanned Aerial Vehicle (UAV) Imagery, Remote Sensing, vol. 4, no. 12, pp. 1573-1599, 2012.

[2] Turner D., Lucieer, A. \& Watson C. An Automated Technique for Generating Georectified Mosaics from Ultra-High Resolution Unmanned Aerial Vehicle (UAV) Imagery, Based on Structure from Motion (SfM) Point Clouds, Remote Sensing, vol. 44 no. 12, pp. 1392-1410, 2012.

[3] Westoby M.J., Brasington J., Glasser N.F., Hambrey M.J. \& Reynolds J.M. Structure-fromMotion photogrammetry: A low-cost, effective tool for geoscience applications, Geomorphology, vol. 179, pp. 300-314, 2012

[4] Wallace L., Lucieer A., Malenovský Z., Turner D. \& Vopěnka, P. Assessment of Forest Structure Using Two UAV Techniques: A Comparison of Airborne Laser Scanning and Structure from Motion Point Clouds, Forests, vol. 7, no. 3, pp. 62, 2016

[5] Surový P., Yoshimoto A. \& Panagiotidis D. Accuracy of Reconstruction of the Tree Stem Surface Using Terrestrial Close-Range Photogrammetry, Remote Sensing, vol. 8, no. 2, pp. 123, 2016. Doi:10.3390/rs8020123

[6] Jensen J. \& Mathews A. Assessment of Image-Based Point Cloud Products to Generate a Bare Earth Surface and Estimate Canopy Heights in a Woodland Ecosystem, Remote Sensing, vol. 8, no. 1, pp. 50, 2016. 
[7] Perks M.T., Russell A.J. \& Large A.R.G. Technical Note: Advances in flash flood monitoring using UAVs, Hydrology and Earth System Sciences Discussions, (February), pp. 1-18, 2016.

[8] Lucieer A., Jong S.M.D. \& Turner D. Mapping landslide displacements using Structure from Motion (SfM) and image correlation of multi-temporal UAV photography, Progress in Physical Geography, vol. 38, no. 1, pp. 97-116, 2014.

[9] Bhardwaj A., Sam L., Akanksha Martín-Torres F.J. \& Kumar R. UAVs as remote sensing platform in glaciology: Present applications and future prospects, Remote Sensing of Environment, vol. 175, pp. 196-204, 2016

[10] Christensen B.R. Use of UAV or remotely piloted aircraft and forward-looking infrared in forest, rural and wildland fire management: evaluation using simple economic analysis, New Zealand Journal of Forestry Science, vol. 45 no. 1, pp. 16, 2015.

[11] Brumana R., Oreni D., Van Hecke L., Barazzetti L., Previtali M., Roncoroni F. \& Valente R. Combined Geometric and Thermal Analysis from UAV Platforms for Archaeological Heritage Documentation, ISPRS Annals of the Photogrammetry, Remote Sensing and Spatial Information Sciences, II(September), pp. 49-54, 2013.

[12] Cunliffe A.M., Brazier R.E. \& Anderson K. Ultra-fine grain landscape-scale quantification of dryland vegetation structure with drone-acquired structure-from-motion photogrammetry, Remote Sensing of Environment, vol. 183, pp. 129-143, 2016.

[13] Bartoš K., Pukanská K. \& Sabová J. The Application Of Open-Source And Free Photogrammetric Software For The Purposes Of Cultural Heritage Documentation, GeoScience Engineering, vol. 60, no. 2, pp. 19-26, 2014.

[14] Santoso F., Garratt M. A., Pickering M. R. \& Asikuzzaman M. 3D Mapping for Visualization of Rigid Structures: A Review and Comparative Study, IEEE Sensors Journal, vol. 16., no. 6, pp. 1484-1507, 2016.

[15] Mierla M., Romanescu G., Nichersu I. \& Grigoras I. Hydrological risk map for the Danube delta - a case study of floods within the fluvial delta, IEEE Journal of Selected Topics in Applied Earth Observations and Remote Sensing, vol. 8, no. 1, pp. 98-104, 2015.

[16] Romanescu G., Cotiuga V., Asandulesei A. \& Stoleriu C. Use of the 3-D scanner in mapping and monitoring the dynamic degradation of soils. Case study of the Cucuteni-Baiceni Gully on the Moldavian Plateau (Romania), Hydrology and Earth System Sciences, vol. 16, pp. 953-966, 2012.

[17] Romanescu G. \& Stoleriu C. Anthropogenic interventions and hydrological-risk phenomena in the fluvial-maritime delta of the Danube (Romania), Ocean \& Coastal Management, vol. 102, pp. 123-130, 2014. 